\title{
On linkages in polytope graphs
}

\author{
Axel Werner and Ronald F. Wotzlaw* \\ (Communicated by M. Henk)
}

\begin{abstract}
A graph is $k$-linked if any $k$ disjoint vertex-pairs can be joined by $k$ disjoint paths. We slightly improve a lower bound on the linkedness of polytopes. This results in exact values for the minimal linkedness of 7-, 10- and 13-dimensional polytopes.

We analyze in detail linkedness of $d$-polytopes on at most $(6 d+7) / 5$ vertices. In that case, a tight lower bound on minimal linkedness is derived, and examples meeting this lower bound are constructed. These examples contain a class of examples due to Gallivan.
\end{abstract}

The Handbook of Discrete and Computational Geometry states the following question by Larman \& Mani [12] as a problem:

Question 1 (Larman \& Mani [12], Kalai [10, Problem 20.2.6]). Let $G$ be the graph of a $d$-polytope and $k=\lfloor d / 2\rfloor$. Is it true that for every two disjoint sequences $\left(s_{1}, \ldots, s_{k}\right)$ and $\left(t_{1}, \ldots, t_{k}\right)$ of vertices of $G$ there are $k$ vertex-disjoint paths connecting $s_{i}$ to $t_{i}$, $i=1, \ldots, k$ ?

This question asks, rephrased in customary graph theory language, whether the graph of every $d$-polytope is $\lfloor d / 2\rfloor$-linked. It was known to Larman \& Mani [12] that the answer is positive in dimensions $d \leq 5$; see also Section 2. Furthermore, in the special case of simplicial polytopes, Larman \& Mani [12] gave a precise answer: Every simplicial $d$ polytope is $\lfloor(d+1) / 2\rfloor$-linked. The stacked polytopes show that this bound cannot be improved.

For general polytopes in higher dimensions the situation is quite different: We discovered polytopes that show that Question 1 has a negative answer in dimensions 8, 10, and $d \geq 12$. Even when $k$ is chosen as $\lfloor 2(d+4) / 5\rfloor$ (which is strictly smaller than $\lfloor d / 2\rfloor$

* Research supported by the Deutsche Forschungsgemeinschaft within the research training group "Methods for Discrete Structures" (GRK 1408). 
for all $d \geq 22$ ), $d$-polytopes are not necessarily $k$-linked. We later learned from Larman [11] that exactly the same polytopes were already discovered in this context in the 1970s by Gallivan [3, Theorem 7, p. 46]. They were later published by McMullen [15] and Gallivan [4].

We denote by $k(d)$ the largest integer such that every $d$-polytope is $k(d)$-linked. Gallivan's examples show that $k(d) \leq\lfloor(2 d+3) / 5\rfloor$, and Larman \& Mani [12] have proven a lower bound of $\lfloor(d+1) / 3\rfloor$. We improve this lower bound marginally to $\lfloor(d+2) / 3\rfloor$ in Section 2. While this improvement is irrelevant for asymptotic questions, it implies exact values for $k(d)$ in dimensions 7,10 , and 13 (previously only the value of $k(8)$ was known in addition to the cases $d \leq 5$; see [4]).

A closer look at Gallivan's examples made it apparent that minimal linkedness of $d$ polytopes on $f_{0}=d+\gamma+1$ vertices does depend on $\gamma$, at least if $\gamma$ is small. We therefore introduce a new parameter $k(d, \gamma)$ that measures minimal linkedness of $d$-polytopes on $d+\gamma+1$ vertices. We determine $k(d, \gamma)$ for polytopes on at most $(6 d+7) / 5$ vertices in Section 3 and analyze the combinatorial types of polytopes with linkedness exactly $k(d, \gamma)$.

We show that among the combinatorial types that meet the lower bound, Gallivan's polytopes are in some sense the canonical ones, in some cases even unique: If $f_{0}$ is odd there is only one combinatorial type with linkedness $k(d, \gamma)$ among all polytopes on $f_{0}$ vertices. This type is given by an iterated pyramid over a join of quadrilaterals. Because of the special combinatorial structure of these polytopes they are even projectively unique; see McMullen [14]. We complement this result by showing that, if $f_{0}$ is even, there are "many" combinatorial types of polytopes with minimal linkedness $k(d, \gamma)$.

One by-product of our analysis is an interesting theorem that classifies polytopes with small facet complements; see Theorem 3.9. We have not seen this characterization in work of others, but suspect that it is known. This characterization implies the well-known characterization of $d$-polytopes on $d+2$ vertices [6, pp. 97-101].

The paper is organized as follows. We introduce basic notions and notation in Section 1. In Section 2, we give lower and upper bounds on $k(d)$. We then study linkedness of polytopes on few vertices in Section 3. We conclude with open problems in Section 4.

Acknowledgements. The authors would like to thank Günter M. Ziegler for helpful discussions on the subject and the anonymous referee for useful suggestions that helped to improve the exposition.

\section{Basic definitions \& notation}

Definition ( $k$-linked, linkage). A graph $G=(V, E)$ is said to be $k$-linked if $|V| \geq 2 k$ and for every choice of $2 k$ distinct vertices $s_{1}, \ldots, s_{k}, t_{1}, \ldots, t_{k}$ there exist $k$ vertex-disjoint paths $L_{1}, \ldots, L_{k}$ such that $L_{i}$ joins $s_{i}$ and $t_{i}$ for $i=1, \ldots, k$. We denote by $k(G)$ the largest integer $k$ such that $G$ is $k$-linked.

If we write $s=\left(s_{1}, \ldots, s_{k}\right)$ and $\boldsymbol{t}=\left(t_{1}, \ldots, t_{k}\right)$, we call the paths $L_{1}, \ldots, L_{k}$ an $(s, t)$-linkage. 
We use the following convenient notation for subpaths of a path $L=v_{0} v_{1} \ldots v_{k}$ (as in [2]),

$$
L v_{i}:=v_{0} \ldots v_{i}, \quad v_{j} L:=v_{j} \ldots v_{k}, \quad v_{j} L v_{i}:=v_{j} \ldots v_{i},
$$

where $j \leq i$ for $i, j \in\{0, \ldots, k\}$. If $L_{1}$ and $L_{2}$ are two paths, we write $L_{1} \cup L_{2}$ for the concatenation of $L_{1}$ and $L_{2}$.

If $P$ is a polytope, we write $G(P)$ for the graph of $P$, that is, the graph defined by incidences between the vertices, denoted $\mathcal{V}(P)$, and edges of $P$. We write $f_{0}$ for the number of vertices of $P$, that is, the cardinality of $\mathcal{V}(P)$. Furthermore, $\operatorname{dim}(P)$ denotes the dimension of $P$. As general references about polytopes, we refer to Grünbaum [6] and Ziegler [21].

Definition (Linkage parameters). We say that a polytope $P$ is $k$-linked if the graph $G(P)$ is $k$-linked and define the following parameters for general polytopes:

$$
\begin{aligned}
k(P) & :=k(G(P)), \\
k(d, \gamma) & :=\min \left\{k(P): P \text { is a } d \text {-polytope with } f_{0}=d+\gamma+1\right\}, \\
k(d) & :=\min _{\gamma} k(d, \gamma) .
\end{aligned}
$$

We call $k(d)$ the minimal linkedness of d-polytopes.

For polytopes $P_{1}$ and $P_{2}$ we write $P_{1} * P_{2}$ for the join of $P_{1}$ and $P_{2}$; see [6, Exercise 4.8.1]. For $P_{2}=\Delta_{n-1}$, that is, the $(n-1)$-simplex, this specializes to the $n$-fold pyramid $\operatorname{pyr}^{n}\left(P_{1}\right)$ over $P_{1}$. If $v$ is a point outside of the affine hull of $P$, we also denote by $\operatorname{pyr}_{v}(P)$ the pyramid over $P$ with apex $v$. Thus, $\operatorname{pyr}_{v}(P)$ is combinatorially isomorphic to $\operatorname{pyr}^{1}(P)$.

For faces $F \subseteq P_{1}$ and $G \subseteq P_{2}$ we denote by $\left(P_{1}, F\right) \oplus\left(P_{2}, G\right)$ the subdirect sum of the polytopes $P_{1}$ and $P_{2}$ with respect to $F$ and $G$; see [14]. This operation specializes to the direct sum of $P_{1}$ and $P_{2}$, denoted by $P_{1} \oplus P_{2}$ if we choose $F=P_{1}$ and $G=P_{2}$; see [6, Exercise 4.8.4].

\section{Minimal linkedness of polytopes}

In this section we provide lower and upper bounds on $k(d)$ for general polytopes in arbitrary dimension $d$.

A graph $G^{\prime}$ is a subdivision of $G=(V, E)$ if $G^{\prime}$ is obtained from $G$ by replacing each edge $u v \in E$ of $G$ by a path with end-vertices $u$ and $v$ (this path may have length one). We call the set of all interior vertices of these paths the subdividing vertices, the other vertices the branch vertices. If a graph $H$ contains a subdivision of a graph $G$, we say that $G$ is a topological minor of $H$. If there is a vertex $v \in V\left(G^{\prime}\right)$ such that the set of branch vertices is $\{v\} \cup U$ with $U \subseteq N(v)$, where $N(v)$ is the set of all neighbors of $v$ in $G$, we say that $G^{\prime}$ is a subdivision of $G$ rooted at $v$. This implies that the vertex in $G$ that corresponds to $v$ is connnected to all other vertices in $G$; we will only apply this definition to the case $G=K_{n}$, the complete graph on $n$ vertices. 
A graph $G=(V, E)$ is $k$-connected if $|V| \geq k+1$, and if for every set $U \subseteq V$ of cardinality at most $k-1$, the graph $G$ cannot be disconnected by removing the vertices in $U$ (and all incident edges).

Besides Balinski's Theorem, which states that every $d$-polytope graph is $d$-connected [1], we need the following theorem of Grünbaum about the structure of $d$-polytope graphs.

Grünbaum's Theorem ([5], [6, Section 11.1, p. 200]). Let $P$ be a d-polytope, $v \in \mathcal{V}(P)$ a vertex of $P$, and $G=G(P)$ the graph of $P$. Then $G$ contains a subdivision of $K_{d+1}$ rooted at $v$.

The original wording of Grünbaum's Theorem is different: It is not mentioned that the subdivision can be chosen rooted at a specified vertex. However, this extension easily follows from Grünbaum's proof.

2.1 Lower bound on minimal linkedness. Larman \& Mani [12] have shown that every $2 k$-connected graph that contains a $K_{3 k}$ as a topological minor is $k$-linked. Robertson $\&$ Seymour [16] have proven the much stronger statement that one may replace "topological minor" by "minor" in the previous statement.

In conjunction with Grünbaum's Theorem this implies that every $d$-polytope is $\lfloor(d+$ $1) / 3$-linked. However, already in dimension 4 this bound is not tight. It is easy to see by a geometric argument that every 4-polytope is 2-linked; this also follows from the characterization of 2-linked graphs by Seymour [17], Shiloach [18], and Thomassen [20], or results by Jung [7].

We improve Larman \& Mani's bound slightly by considering subdivisions of complete graphs that are rooted at a specified vertex. This new bound directly implies that 4polytopes are 2-linked.

The proof of the following lemma is a variation of an argument by Diestel [2, pp. 7071].

Lemma 2.1. Let $G=(V, E)$ be a $2 k$-connected graph. Suppose that for every vertex $v$ of $G$ the graph $G$ contains a subdivision of $K_{3 k-1}$ rooted at $v$. Then $G$ is $k$-linked.

Proof. Let $s=\left(s_{1}, \ldots, s_{k}\right)$ and $\boldsymbol{t}=\left(t_{1}, \ldots, t_{k}\right)$, where $s_{1}, \ldots, s_{k}, t_{1}, \ldots, t_{k}$ are distinct vertices of $G$. Let $K$ be a subdivision of $K_{3 k-1}$ rooted at vertex $t_{k}$ with branch vertices $U:=U^{\prime} \cup\left\{t_{k}\right\}$, where $U^{\prime} \subseteq N\left(t_{k}\right)$.

Since $G \backslash\left\{t_{k}\right\}$ is $(2 k-1)$-connected there exist $2 k-1$ disjoint paths $S_{1}, \ldots, S_{k}$ and $T_{1}, \ldots, T_{k-1}$ in $G$ that avoid $t_{k}$ such that $S_{i}$ joins $s_{i}$ to $U^{\prime}$, for $i=1, \ldots, k$, and $T_{i}$ joins $t_{i}$ to $U^{\prime}$, for $i=1, \ldots, k-1$. Moreover, we assume that the paths have been chosen such that they do not have interior vertices in $U^{\prime}$ (and thus also not in $U$ ) and that their total number of edges outside of the set of edges $E(K)$ is minimal.

Let $W=\left\{v_{1}, \ldots, v_{k}, w_{1}, \ldots, w_{k-1}\right\}$ be the vertices of these paths in $U^{\prime}$, where $v_{i}$ is in $S_{i}$ and $w_{i}$ is in $T_{i}$. We then have a partition of $U$ into sets $\left\{t_{k}\right\}, W$ and $W^{\prime}:=U^{\prime} \backslash W$ with $\left|W^{\prime}\right|=k-1$. Let $u_{1}, \ldots, u_{k-1}$ be the vertices in $W^{\prime} \subseteq U$. We call these vertices free. 
Since the path $S_{k}$ joins $s_{k}$ to a neighbor of $t_{k}$ the path $L_{k}:=S_{k} \cup T_{k}$ joins $s_{k}$ and $t_{k}$, where $T_{k}$ is the path that consists of the single edge from the vertex $s_{k}$ to the vertex $t_{k}$.

Now fix some $i \in\{1, \ldots, k-1\}$ and let $M_{i}$ be the path in $K$ from the free vertex $u_{i}$ to $v_{i}$ and $N_{i}$ be the path in $K$ from $u_{i}$ to $w_{i}$. Since the paths $S_{1}, \ldots, S_{k}, T_{1}, \ldots, T_{k-1}$ were chosen minimal with respect to their number of edges outside of $K$ and $u_{i}$ is a free vertex, the paths $S_{j}$ are disjoint from $M_{i}$ for $j \neq i$, and they are disjoint from $N_{i}$ for all $j=1, \ldots, k$. Similarly, the paths $T_{j}$ are disjoint from $N_{i}$ for $j \neq i$, and they are disjoint from $M_{i}$ for all $j=1, \ldots, k-1$. Hence we can join $v_{i}$ to $w_{i}$ via the free vertex $u_{i}$.

Denote by $s_{i}^{\prime}$ the intersection vertex of $S_{i}$ and $M_{i}$ that is closest to $u_{i}$, and by $t_{i}^{\prime}$ the intersection vertex of $T_{i}$ and $N_{i}$ that is closest to $u_{i}$. We then get pairwise disjoint paths

$$
L_{i}= \begin{cases}\left(S_{i} s_{i}^{\prime}\right) \cup\left(s_{i}^{\prime} M_{i}\right) \cup\left(N_{i} t_{i}^{\prime}\right) \cup\left(t_{i}^{\prime} T_{i}\right), & 1 \leq i \leq k-1 \\ S_{k} \cup T_{k}, & i=k\end{cases}
$$

such that $L_{i}$ joins $s_{i}$ and $t_{i}$, that is, an $(\boldsymbol{s}, \boldsymbol{t})$-linkage.

Theorem 2.2. Every d-polytope is $\lfloor(d+2) / 3\rfloor$-linked.

Proof. Let $P$ be a $d$-polytope. We set $k:=\lfloor(d+2) / 3\rfloor$. For $d \geq 2$, we then have $d \geq 2 k$ and $d+1 \geq 3 k-1$. Therefore, by Grünbaum's Theorem the graph $G(P)$ contains, at every vertex $v$, a $K_{3 k-1}$ subdivision rooted at $v$. By Balinski's Theorem, $G(P)$ is at least $2 k$-connected. Lemma 2.1 implies that the graph of $P$ is $k$-linked.

\subsection{Upper bound on minimal linkedness.}

Theorem 2.3. Let $d \geq 2$ and $\gamma \geq 1$. Then the minimal linkedness of $d$-polytopes on $d+\gamma+1$ vertices satisfies

$$
k(d, \gamma) \leq\lfloor d / 2\rfloor .
$$

Proof. For $d=2$ the assertion clearly is true. Let $d \geq 3$ and $\gamma \geq 1$. To prove the statement we have to construct a $d$-polytope on $d+\gamma+1$ vertices with $k(P) \leq\lfloor d / 2\rfloor$.

Let $Q$ be a 3-polytope on $4+\gamma$ vertices that has a square facet. For instance, for $\gamma=1$ take the pyramid over a square and for $\gamma>1$ stack this pyramid $\gamma-1$ times over triangular facets. Let $P:=\operatorname{pyr}^{d-3}(Q)$, the $(d-3)$-fold pyramid over $Q$. Then $P$ is a $d$-polytope, has $d+\gamma+1$ vertices, and is not $(\lfloor d / 2\rfloor+1)$-linked. To see this let $s_{1}, t_{1}, s_{2}, t_{2}$ be the vertices of a square facet of $Q$ (in that order around the facet). Then, by planarity, these cannot be linked in $G(Q)$.

Additionally, with $m:=\lfloor(d-3) / 2\rfloor$ there are exactly $2 m$ vertices in $\mathcal{V}(P) \backslash \mathcal{V}(Q)$ if $d$ is odd and exactly $2 m+1$ if $d$ is even. We choose distinct vertices $s_{3}, \ldots, s_{m+2}, t_{3}, \ldots$, $t_{m+2}$ arbitrarily from the set $\mathcal{V}(P) \backslash \mathcal{V}(Q)$ and, if $d$ is even, we let $s_{m+3}$ be the last vertex left in $\mathcal{V}(P) \backslash \mathcal{V}(Q)$ and choose $t_{m+3}$ arbitrarily from $\mathcal{V}(Q) \backslash\left\{s_{1}, s_{2}, t_{1}, t_{2}\right\}$.

This set of $\lfloor d / 2\rfloor+1$ pairs of vertices cannot be linked in $P$. Therefore $k(P) \leq$ $\lfloor d / 2\rfloor$.

In the case $\gamma=0$ we trivially have $k(d, \gamma)=\lfloor(d+1) / 2\rfloor$, as the $d$-simplex is $\lfloor(d+1) / 2\rfloor$-linked. Theorem 2.3 implies that $k(d) \leq\lfloor d / 2\rfloor$. We improve this bound significantly in the next section. 


\section{Linkages in polytopes with few vertices}

In this section, we study linkedness of $d$-polytopes with $f_{0}=d+\gamma+1$ vertices, where $\gamma$ is small. More precisely, for $\gamma \leq(d+2) / 5$ we determine the value of $k(d, \gamma)$ and analyze polytopes that attain the value of $k(d, \gamma)$.

3.1 Lower bound for polytopes with few vertices. The linkedness of a graph is a local property in the following sense: If a graph is highly connected, then a $k$-linked subgraph ensures $k$-linkedness for the whole graph. The precise statement is the following lemma.

Lemma 3.1. Let $G=(V, E)$ be a $2 k$-connected graph and $G^{\prime}$ a subgraph of $G$ that is $k$-linked. Then $G$ is k-linked.

Proof. Let $s=\left(s_{1}, \ldots, s_{k}\right)$ and $\boldsymbol{t}=\left(t_{1}, \ldots, t_{k}\right)$, where $s_{1}, \ldots, s_{k}, t_{1}, \ldots, t_{k}$ are distinct vertices in $G$. Since $G$ is $2 k$-connected, there exist $2 k$ vertex disjoint paths $S_{1}, \ldots, S_{k}$ and $T_{1}, \ldots, T_{k}$ such that $S_{i}$ connects $s_{i}$ to $G^{\prime}$ and $T_{i}$ connects $t_{i}$ to $G^{\prime}$. We choose the paths such that each contains only one vertex from $G^{\prime}$. Let $\left\{s_{i}^{\prime}\right\}=G^{\prime} \cap S_{i}$ and $\left\{t_{i}^{\prime}\right\}=G^{\prime} \cap T_{i}$, for $i=1, \ldots, k$.

Since $G^{\prime}$ is $k$-linked there exists an $\left(\boldsymbol{s}^{\prime}, \boldsymbol{t}^{\prime}\right)$-linkage $L_{1}^{\prime}, \ldots, L_{k}^{\prime}$ in $G^{\prime}$ for $\boldsymbol{s}^{\prime}=\left(s_{1}^{\prime}, \ldots\right.$, $\left.s_{k}^{\prime}\right)$ and $\boldsymbol{t}^{\prime}=\left(t_{1}^{\prime}, \ldots, t_{k}^{\prime}\right)$. Then

$$
L_{i}=S_{i} \cup L_{i}^{\prime} \cup T_{i}, \quad 1 \leq i \leq k
$$

is an $(s, t)$-linkage in $G$.

We obtain a lower bound on linkedness of polytopes with few vertices by finding a highly-linked subgraph in the graph of $P$. This highly-linked subgraph is a complete subgraph: the graph of a simplex face of high dimension.

Lemma 3.2 (Kalai [9], Marcus [13]). Let $P$ be a d-polytope on $d+\gamma+1$ vertices with $d \geq \gamma$. Then $P$ has a $(d-\gamma)$-face that is a simplex.

Proof. One checks easily that the statement is true for every 2-polytope on $3+\gamma$ vertices, $\gamma \geq 0$. Let $P$ be a $d$-polytope, $d \geq 3$. Choose a facet $F$, which is of dimension $d^{\prime}=d-1$. Suppose that $F$ has $d^{\prime}+\gamma^{\prime}+1$ vertices, where $0 \leq \gamma^{\prime} \leq \gamma$. By induction, $F$ has a simplex face $S$ of dimension $\operatorname{dim}(S)=d^{\prime}-\gamma^{\prime}=d-1-\gamma^{\prime}=d-\left(\gamma^{\prime}+1\right)$. If $\gamma \geq \gamma^{\prime}+1$, then $\operatorname{dim}(S) \geq d-\gamma$ and we are done. If $\gamma=\gamma^{\prime}$, then $\mathcal{V}(P) \backslash \mathcal{V}(F)=\{v\}$ and $P=\operatorname{pyr}_{v}(F)$, that is, $P$ is a pyramid over $F$ with apex $v$. Hence $\operatorname{pyr}_{v}(S)$ is a face of $P$ and a simplex of $\operatorname{dimension} \operatorname{dim}(S)+1=d-\gamma$.

Lemma 3.3. Let $d \geq 2$, and $\gamma \geq 0$ with $d \geq \gamma$. Then

$$
k(d, \gamma) \geq\left\lfloor\frac{d-\gamma+1}{2}\right\rfloor .
$$

Proof. In the special case $\gamma=0$ the assertion is trivially true. For $d \geq 2, \gamma \geq 1$ it follows from Lemma 3.1 and Lemma 3.2, since $2\lfloor(d-\gamma+1) / 2\rfloor \leq d-\gamma+1 \leq d$, and the graph of a $d$-polytope is at least $d$-connected, by Balinski's Theorem. 
3.2 Upper bound for polytopes with few vertices. To prove a good upper bound on the number $k(d, \gamma)$ we have to find a polytope $P$ on $d+\gamma+1$ vertices with small $k(P)$. For $\gamma \leq(d+2) / 5$ the lower bound of Lemma 3.3 can be attained.

The examples we describe here were first discovered in this context by Gallivan [3] [4]. He constructed them using Gale diagrams.

In the following, we denote by $\square$ the 2-dimensional cube, that is, the (combinatorially unique) 2-polytope on 4 vertices.

Definition. For integers $n, m \geq 0$ and $j_{1}, k_{1}, \ldots, j_{m}, k_{m} \geq 1$ define

$$
\mathcal{G}\left(n, j_{1}, k_{1}, \ldots, j_{m}, k_{m}\right):=\Delta_{n-1} *\left(\Delta_{j_{1}} \oplus \Delta_{k_{1}}\right) * \cdots *\left(\Delta_{j_{m}} \oplus \Delta_{k_{m}}\right)
$$

and

$$
\mathcal{G}(n, m):=\mathcal{G}(n, \underbrace{1, \ldots, 1}_{2 m \text { times }})=\Delta_{n-1} * \underbrace{\square * \cdots * \square}_{m \text { factors }} .
$$

We also write

$$
\Delta_{n-1} * \square^{* m}:=\Delta_{n-1} * \underbrace{\square * \cdots * \square}_{m \text { factors }} .
$$

The parameters $d$ and $\gamma$ for $\mathcal{G}(n, m)$ can be determined by observing that $\mathcal{G}(n, m)$ has $4 m+n$ vertices, so $d+\gamma+1=4 m+n$, and dimension $\operatorname{dim}(\mathcal{G}(n, m))=n-1+3 m$. Therefore we have

$$
\begin{aligned}
& d=n-1+3 m, \quad \text { and } \\
& \gamma=m .
\end{aligned}
$$

We consider the complement graph $\bar{G}(\mathcal{G}(n, m))$ of $G(\mathcal{G}(n, m))$ in order to examine the linkedness of the polytopes $\mathcal{G}(n, m)$. It is easy to see that the graph of the join of two polytopes corresponds to the join of the graphs of the polytopes. Thus, the complement graph $\bar{G}(\mathcal{G}(n, m))$ has the following form.

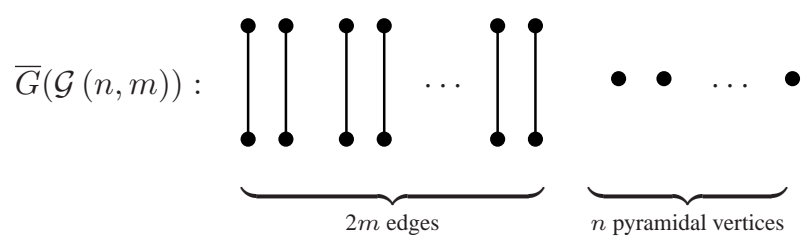

The reason for the low linkedness of $\mathcal{G}(n, m)$ is that there are few vertices that can be used on a "detour" for a linkage between the $2 m$ pairs that are not connected by an edge.

To determine the linkedness of $\mathcal{G}(n, m)$, we determine the linkedness of graphs of type $K_{p} \backslash M$, where $M$ is a matching. Obviously, the graphs $G(\mathcal{G}(n, m))$ are of this type. 
Lemma 3.4. Let $G=K_{p} \backslash M$, where $M$ is a matching of size $q \leq p / 2$, that is, $\bar{G}$ consists of $q$ disjoint edges and $p-2 q$ isolated vertices. Then the linkedness of $G$ is

$$
k(G)= \begin{cases}\left\lfloor\frac{p}{3}\right\rfloor, & \text { if } p \leq 3 q-1 \\ \left\lfloor\frac{p-q}{2}\right\rfloor, & \text { if } p \geq 3 q-1 .\end{cases}
$$

Proof. Observe that the connectivity $\kappa(G)$, that is, the largest integer $k$ such that $G$ is $k$-connected, is given by

$$
\kappa(G)= \begin{cases}p, & \text { if } q=0 \\ p-1, & \text { if } q \geq 1,\end{cases}
$$

and that $G$ has a complete subgraph of size $p-q$. By Lemma 3.1, we thus have

$$
k(G) \geq \min \left\{\left\lfloor\frac{\kappa(G)}{2}\right\rfloor,\left\lfloor\frac{p-q}{2}\right\rfloor\right\} .
$$

Let $p \geq 3 q-1$. Then choose all $q$ pairs of nonconnected vertices and additional $\left\lfloor\frac{p-3 q+2}{2}\right\rfloor$ pairs arbitrarily from the vertices of full degree. Then these cannot be linked in $G$ and the linkedness satisfies

$$
k(G) \leq q+\left\lfloor\frac{p-3 q}{2}\right\rfloor=\left\lfloor\frac{p-q}{2}\right\rfloor .
$$

For $q \geq 1$, we have $\lfloor(p-q) / 2\rfloor \leq\lfloor\kappa(G) / 2\rfloor$, and for $q=0$, we have $\lfloor\kappa(G) / 2\rfloor=\lfloor p / 2\rfloor$, and thus Inequality (3) implies that also $k(G) \geq\lfloor(p-q) / 2\rfloor$.

Let $p \leq 3 q-1$. Then choose $\lfloor p / 3\rfloor+1$ of the $q$ nonedges of $G$ as pairs. These cannot be linked in $G$, as there are at most $p-2(\lfloor p / 3\rfloor+1) \leq\lfloor p / 3\rfloor$ additional vertices. Clearly, any $\lfloor p / 3\rfloor$ pairs of vertices of $G$ can be linked, as any such pair needs at most one additional vertex to be connected, and if a pair is a nonedge, then every other vertex can be used.

For $p=3 q-1$, both terms yield the same value.

Lemma 3.5. Let $n, m \geq 0$ be integers. The linkedness of $\mathcal{G}(n, m)$ is given by

$$
k(\mathcal{G}(n, m))= \begin{cases}\left\lfloor\frac{4 m+n}{3}\right\rfloor, & \text { if } n \leq 2 m-1 \\ \left\lfloor\frac{2 m+n}{2}\right\rfloor, & \text { if } n \geq 2 m-1 .\end{cases}
$$

If we use substitutions (1) and (2), this evaluates to

$$
k(\mathcal{G}(n, m))= \begin{cases}\left\lfloor\frac{d+\gamma+1}{3}\right\rfloor, & \text { if } d+2 \leq 5 \gamma \\ \left\lfloor\frac{d-\gamma+1}{2}\right\rfloor, & \text { if } d+2 \geq 5 \gamma .\end{cases}
$$

Proof. This follows from Lemma 3.4, as the number of vertices of $\mathcal{G}(n, m)$ is $4 m+n$ and the number of nonedges is $2 \mathrm{~m}$. 
Example 1. Let $d=8$ and $\gamma=2$. Then $n=3$ and $m=2$ and we obtain the 8-polytope on 11 vertices

$$
P:=\mathcal{G}(3,2)=\Delta_{2} * \square * \square=\operatorname{pyr}^{3}(\square * \square)
$$

The complement of the graph of $P$ consists of 4 disjoint edges and 3 isolated vertices:

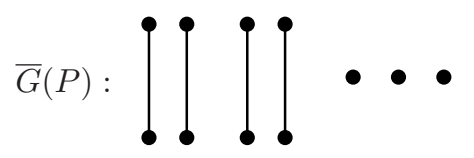

The four pairs of vertices corresponding to the four nonedges of $G(P)$ cannot be linked in $G(P)$, because there are only three additional vertices. Thus, the polytope $P$ is not 4-linked. It is the smallest known example of a polytope that is not $\lfloor d / 2\rfloor$-linked.

In combination with Lemma 3.3 we obtain the following result.

Theorem 3.6. Let $d \geq 2$ and $0 \leq \gamma \leq(d+2) / 5$. Then

$$
k(d, \gamma)=\left\lfloor\frac{d-\gamma+1}{2}\right\rfloor
$$

Choosing $\gamma=\lfloor(d+2) / 5\rfloor$, we obtain Gallivan's examples, and the bound of the last theorem implies the following bound on $k(d)$ first given by Gallivan [4].

Corollary 3.7 (Gallivan [4]). The minimal linkedness of d-polytopes satisfies

$$
k(d) \leq\lfloor(2 d+3) / 5\rfloor
$$

3.3 Polytopes that meet the lower bound. The main theorem of this section is Theorem 3.11. It states that polytopes that meet the lower bound of Lemma 3.3 are

- unique if $f_{0}$ is odd, and

- of rather restricted combinatorial type, otherwise.

Furthermore, a lower bound on the number of combinatorial types in the latter case is proven.

The "road map" to Theorem 3.11 is the following: We prove a concise characterization of polytopes that have small facet complements in Theorem 3.9. This theorem already imposes severe conditions on the combinatorial types of polytopes that meet the lower bound. Afterwards we analyze the graphs of the types that appear in Theorem 3.9, which yields a further restriction; see Lemma 3.10.

The proof of Theorem 3.11 then mainly consists of the construction of combinatorial types for the case when $f_{0}$ is even. 
3.3.1 Polytopes with small facet complements. We consider polytopes with small facet complements, that is, polytopes in which every facet contains almost all vertices. If the size of every facet complement is one, then clearly the polytope in question is the simplex.

The following lemma is the next step: What combinatorial types are possible if for every facet there are at most 2 vertices that are not contained in that facet?

In the following, if $P$ is a polytope and $F$ a face of $P$, we denote by $P / F$ the face figure of $P$ at $F$; see [21, Chapter 2]. The face lattice of $P / F$ is the interval from $F$ to $P$ in the face lattice of $P$.

Lemma 3.8. Let $P$ be a d-polytope such that every facet $F$ of $P$ satisfies the inequality $|\mathcal{V}(P) \backslash \mathcal{V}(F)| \leq 2$. Then $P$ is of the form

$$
\mathcal{G}\left(n, j_{1}, k_{1}, \ldots, j_{m}, k_{m}\right)=\Delta_{n-1} *\left(\Delta_{j_{1}} \oplus \Delta_{k_{1}}\right) * \cdots *\left(\Delta_{j_{m}} \oplus \Delta_{k_{m}}\right)
$$

where $k_{1}, \ldots, k_{m}, j_{1}, \ldots, j_{m} \geq 1$, and $d=n-1+j_{1}+k_{1}+\cdots+j_{m}+k_{m}+m$.

Proof. The property $|\mathcal{V}(P) \backslash \mathcal{V}(F)| \leq 2$ implies that the hypergraph of facet-complements, that is, the hypergraph

$$
G_{\text {cofacet }}(P):=(\mathcal{V}(P),\{W \subseteq \mathcal{V}(P): \mathcal{V}(P) \backslash W \text { is the vertex set of a facet }\})
$$

is a graph (with no parallel edges, but possibly with loops). The edges of $G_{\text {cofacet }}(P)$ are in bijection with the facets of $P$. Since the combinatorial type of a polytope is determined by the vertex-facet incidences, the combinatorial type of $G_{\text {cofacet }}(P)$ determines the combinatorial type of $P$.

For $Q=\mathcal{G}\left(n, j_{1}, k_{1}, \ldots, j_{m}, k_{m}\right)$ the graph $G_{\text {cofacet }}(Q)$ is a disjoint union of $n$ copies of the graph that consists of one single vertex and one single loop, and complete bipartite graphs $K_{j_{1}, k_{1}}, \ldots, K_{j_{m}, k_{m}}$ (the complete bipartite graph $K_{\ell, \ell^{\prime}}$ consists of two independent sets of cardinalities $\ell$ and $\ell^{\prime}$, respectively, and all edges in between them). Thus it suffices to show that $G_{\text {cofacet }}(P)$ is of this type.

It is easy to see that loops can only occur at isolated vertices and that there are no vertices of degree 1 in $G_{\text {cofacet }}(P)$ (we follow the convention that loops contribute two edges to the degree count). Then it suffices to check the following two properties of $G_{\text {cofacet }}(P)$ :

(i) The graph $G_{\text {cofacet }}(P)$ does not have odd cycles (except for loops at isolated vertices).

(ii) Whenever there is a path $v_{1} v_{2} v_{3} v_{4}$ of length 3 in $G_{\text {cofacet }}(P)$, then $v_{1} v_{4}$ is also an edge of $G_{\text {cofacet }}(P)$.

In fact, (i) follows from (ii) and the absence of triangles, as any larger odd cycle together with (ii) would imply the existence of a triangle.

We now show that $G_{\text {cofacet }}(P)$ does not have triangles. Suppose there is a triangle with vertices $v_{1}, v_{2}, v_{3}$ and edges corresponding to facets $F_{1}, F_{2}, F_{3}$ with $\mathcal{V}\left(F_{1}\right)=\mathcal{V}(P) \backslash$ $\left\{v_{2}, v_{3}\right\}, \mathcal{V}\left(F_{2}\right)=\mathcal{V}(P) \backslash\left\{v_{1}, v_{3}\right\}$, and $\mathcal{V}\left(F_{3}\right)=\mathcal{V}(P) \backslash\left\{v_{1}, v_{2}\right\}$. Let $F^{\prime}$ be the face $F_{1} \cap F_{2}=F_{1} \cap F_{3}=F_{2} \cap F_{3}$. Then clearly $F_{1}=\operatorname{pyr}_{v_{4}}\left(F^{\prime}\right), F_{2}=\operatorname{pyr}_{v_{2}}\left(F^{\prime}\right)$, and $F_{3}=$

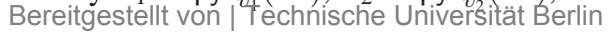


$\operatorname{pyr}_{v_{3}}\left(F^{\prime}\right)$. Thus $\operatorname{dim} F^{\prime}=d-2$ and $P / F^{\prime}$ is a 1 -polytope on 3-vertices, a contradiction. (In face lattice terms, this is a contradiction to the diamond property.)

Finally, we show that a path $v_{1} v_{2} v_{3} v_{4}$ of length 3 implies the existence of the edge $v_{1} v_{4}$. The edges of the path $v_{1} v_{2} v_{3} v_{4}$ correspond to facets $F_{1}, F_{2}$, and $F_{3}$ with $\mathcal{V}\left(F_{1}\right)=$ $\mathcal{V}(P) \backslash\left\{v_{1}, v_{2}\right\}, \mathcal{V}\left(F_{2}\right)=\mathcal{V}(P) \backslash\left\{v_{2}, v_{3}\right\}$, and $\mathcal{V}\left(F_{3}\right)=\mathcal{V}(P) \backslash\left\{v_{3}, v_{4}\right\}$. Let $F^{\prime}=$ $F_{1} \cap F_{2} \cap F_{3}$. Then clearly $F^{\prime}$ has dimension $d-3$, as only four vertices of $P$ do not lie on $F^{\prime}$.

The facets $F_{1}, F_{2}$ and $F_{3}$ are of dimension $d-1$ and each of them contains exactly two vertices more than $F^{\prime}$. We conclude that $\operatorname{pyr}_{v_{1}}\left(F^{\prime}\right), \operatorname{pyr}_{v_{2}}\left(F^{\prime}\right), \operatorname{pyr}_{v_{3}}\left(F^{\prime}\right)$, and $\operatorname{pyr}_{v_{4}}\left(F^{\prime}\right)$ are all faces of $P$. Thus, $P / F^{\prime}$ is a 2-polytope on 4 vertices, which implies that $F_{4}:=$ $\operatorname{pyr}_{v_{3}}\left(\operatorname{pyr}_{v_{2}}\left(F^{\prime}\right)\right)$ is also a facet of $P$ with $\mathcal{V}\left(F_{4}\right)=\mathcal{V}(P) \backslash\left\{v_{1}, v_{4}\right\}$.

Theorem 3.9. Let $d \geq 2$, let $0 \leq \gamma \leq d-2$, and let $P$ be a d-polytope on $d+\gamma+1$ vertices. Then the following are equivalent:

(i) Every facet $F$ of $P$ satisfies $|\mathcal{V}(P) \backslash \mathcal{V}(F)| \leq 2$

(ii) With $m=\gamma$, the polytope $P$ is of the form

$$
\mathcal{G}\left(n, j_{1}, k_{1}, \ldots, j_{m}, k_{m}\right)=\Delta_{n-1} *\left(\Delta_{j_{1}} \oplus \Delta_{k_{1}}\right) * \cdots *\left(\Delta_{j_{m}} \oplus \Delta_{k_{m}}\right)
$$

with suitable parameters $j_{i}$ and $k_{i}$.

(iii) The polytope $P$ does not have a simplex face of dimension $d-\gamma+1$.

Proof. If $|\mathcal{V}(P) \backslash \mathcal{V}(F)| \leq 2$ for every facet $F$ of $P$, then by Lemma 3.8 the polytope $P$ is of the form $\mathcal{G}\left(n, j_{1}, k_{1}, \ldots, j_{m}, k_{m}\right)$. Clearly, we have that $m=\gamma$.

Now, suppose $P$ is an iterated pyramid over a join of sums of simplices. Let $S$ be a simplex face of $P$ of maximal dimension. Then $S$ is the join of $\Delta_{n-1}$ with facets from each factor $\Delta_{j_{i}} \oplus \Delta_{k_{i}}$. A facet of this sum in turn is obtained by leaving out a vertex from each of the two simplices. Hence, $S$ has

$$
n+j_{1}+k_{1}+\cdots+j_{m}+k_{m}=d-m+1=d-\gamma+1
$$

vertices and therefore dimension $d-\gamma$.

Finally, if $P$ does not have a simplex face of dimension $d-\gamma+1$, then $|\mathcal{V}(P) \backslash \mathcal{V}(F)| \leq$ 2 for every facet $F$. Otherwise, suppose there is a facet $F$ with $|\mathcal{V}(P) \backslash \mathcal{V}(F)| \geq 3$. Then $\gamma(F) \leq \gamma-2$, and by Lemma 3.2 the facet $F$ has a simplex face of dimension

$$
(d-1)-\gamma(F)=d-(\gamma(F)+1) \geq d-\gamma+1
$$

in contradiction to the hypothesis.

Theorem 3.9 contains the classification of $d$-polytopes on $d+2$ vertices; see Grünbaum [6, pp. 97-101]: No $d$-polytope on $d+2$ vertices contains a simplex $d$-face. Thus, all polytopes on $d+2$ vertices are of type $\mathcal{G}\left(n, j_{1}, k_{1}, \ldots, j_{m}, k_{m}\right)$ with $m=\gamma=1$. 
3.3.2 Polytopes without large complete subgraphs. So far, we have only excluded large simplex faces. The following lemma analyzes the situation if we also exclude large complete subgraphs.

Lemma 3.10. Let $P$ be a d-polytope on $d+\gamma+1$ vertices. Suppose that the graph $G(P)$ does not have a $K_{d-\gamma+2}$-subgraph. Then $P$ is of the form

$$
\mathcal{G}(n, m)=\Delta_{n-1} * \underbrace{\square * \cdots * \square}_{m \text { factors }},
$$

with $n=d-3 \gamma+1$ and $m=\gamma$.

Proof. Since $P$ does not have a $K_{d-\gamma+2}$-subgraph, $P$ does not have a simplex face of dimension $d-\gamma+1$. Thus, by Theorem 3.9, $P$ is of the form $\mathcal{G}\left(n, j_{1}, k_{1}, \ldots, j_{m}, k_{m}\right)$.

To show that $j_{1}=k_{1}=\cdots=j_{m}=k_{m}=1$ observe that the graph

$$
G\left(\Delta_{j} \oplus \Delta_{k}\right) \begin{cases}\text { is the complete graph } K_{j+k+2} & \text { if } j, k \geq 2 \\ \text { contains a } K_{j+k+1} & \text { if } j \geq 2, k=1 \text { or } j=1, k \geq 2 \\ \text { is a 4-cycle } & \text { if } j=k=1 .\end{cases}
$$

Furthermore, in a join $P * Q$ every vertex of $P$ defines an edge with every vertex of $Q$. Suppose now that $j_{i} \geq 2$ or $k_{i} \geq 2$ for some $i$. Then $G(P)$ contains a complete graph on

$$
n+j_{1}+k_{1}+\cdots+j_{i}+k_{i}+1+\cdots+j_{m}+k_{m}=d-m+2=d-\gamma+2
$$

vertices, which contradicts the hypothesis.

\subsubsection{Combinatorial types that meet the lower bound.}

Theorem 3.11. Let $d \geq 2$, and $0 \leq \gamma \leq(d+1) / 3$. Set $n:=d-3 \gamma+1 \geq 0$, and $m:=\gamma$. Let $P$ be a d-polytope on $f_{0}=d+\gamma+1$ vertices with linkedness $k(P)=\lfloor(d-\gamma+1) / 2\rfloor$.

If $f_{0}$ is odd, then $P$ is of type

$$
\mathcal{G}(n, m)=\Delta_{n-1} * \underbrace{\square * \cdots * \square}_{m \text { factors }} .
$$

If $f_{0}$ is even, then there are at least $2 d-3 \gamma+1$ possibilities for the combinatorial type of $P$. More precisely, exactly one of the following cases applies:

(i) The polytope $P$ is of type $\mathcal{G}(n, m)$.

(ii) We have $m \geq 1$ and $P$ is of type

$$
\mathcal{G}(n-k, \underbrace{1, \ldots, 1}_{2 m-1 \text { times }}, k+1)
$$

with $1 \leq k \leq n$. In this case there are exactly $n=d-3 \gamma+1$ possibilities for the combinatorial type of $P$. 
(iii) We have $m \geq 2$ and $P$ has a facet $F$ of type

$$
\Delta_{n+4} * \underbrace{\square * \cdots * \square}_{m-2 \text { factors }} .
$$

In particular, $k(F)=k(P)$. In this case there are at least $d-1$ possibilities for the combinatorial type of $P$.

Proof. If $P$ is a $d$-polytope with $d \geq 2$ on $d+\gamma+1$ vertices and $k(P)=\lfloor(d-\gamma+1) / 2\rfloor$, then clearly $d>\gamma \geq 0$, as every such polytope is at least 1-linked.

Let $f_{0}$ be odd, that is, $d-\gamma$ is even. If $k(P)=\lfloor(d-\gamma+1) / 2\rfloor$, then $G(P)$ cannot have a $K_{d-\gamma+2}$-subgraph, and by Lemma 3.10 the polytope $P$ is of type $\mathcal{G}(n, m)$ with $m=\gamma$ and $n=d+\gamma+1-4 \gamma=d-3 \gamma+1$.

Let $f_{0}$ be even, that is, $d-\gamma$ is odd. Under this assumption, the graph of $P$ cannot have a $K_{d-\gamma+3}$-subgraph. Otherwise its linkedness would be larger than $\lfloor(d-\gamma+1) / 2\rfloor$. We distinguish the following three cases:

(1) The graph $G(P)$ does not have a $K_{d-\gamma+2}$-subgraph.

(2) The graph $G(P)$ does have a $K_{d-\gamma+2}$-subgraph, but $P$ does not have a $(d-\gamma+1)$ dimensional simplex face.

(3) The polytope $P$ has a $(d-\gamma+1)$-simplex face.

These are all possible cases, and clearly they exclude each other.

Case (1). In this case, Lemma 3.10 implies that $P$ is of type $\mathcal{G}(n, m)$.

Case (2). Under the assumptions in (2), Theorem 3.9 implies that the polytope $P$ is of type $\mathcal{G}\left(n^{\prime}, j_{1}, k_{1}, \ldots, j_{m}, k_{m}\right)$ for $m=\gamma \geq 1$ and suitable parameters $n^{\prime}, j_{1}, k_{1}, \ldots, j_{m}$, $k_{m}$.

The complement graph $\bar{G}(P)$ consists of some number $q$ of disjoint edges and $f_{0}-2 q$ isolated vertices. Since $G(P)$ does not have a $K_{d-\gamma+3}$ subgraph we must have $f_{0}-q \leq$ $d-\gamma+2$, that is,

$$
q \geq 2 \gamma-1
$$

The only polytope of type $\mathcal{G}\left(n^{\prime}, j_{1}, k_{1}, \ldots, j_{m}, k_{m}\right)$ with $2 \gamma$ disjoint edges, which is the maximum possible value for $q$, is $\mathcal{G}(n, m)$. However, $\mathcal{G}(n, m)$ does not have a $(d-\gamma+1)$-simplex face and thus we have $q=2 \gamma-1$. This is only achievable if, up to symmetry, $j_{1}=k_{1}=\cdots=j_{m-1}=k_{m-1}=1, j_{m}=1$ and $2 \leq k_{m} \leq d-3 \gamma+2$, that is, the first $m-1$ factors of the join are quadrilaterals and the last factor is a bipyramid over a $k_{m}$-simplex. Thus $P$ is of type

$$
\mathcal{G}(n-k, \underbrace{1, \ldots, 1}_{2 m-1 \text { times }}, k+1),
$$

for $1 \leq k \leq n=d-3 \gamma+1$. This gives $d-3 \gamma+1$ different combinatorial types for the different choices of $k$.

Case (3). In the final case, the polytope $P$ has a $(d-\gamma+1)$-simplex face but not a $(d-\gamma+2)$-simplex face. 
Then for every facet $F$ of $P$ we have $|\mathcal{V}(P) \backslash \mathcal{V}(F)| \leq 3$. Otherwise there is a facet $F^{\prime}$, which is of dimension $d-1$, that has $\gamma\left(F^{\prime}\right) \leq \gamma-3$. That is, $F^{\prime}$ contains a simplex face of dimension $(d-1)-(\gamma-3)=d-\gamma+2$, in contradiction to the hypothesis.

By Theorem 3.9, there exists a facet $F$ with $|\mathcal{V}(P) \backslash \mathcal{V}(F)|=3$, since otherwise $P$ would not have a $(d-\gamma+1)$-simplex face. This facet must satisfy $\left|\mathcal{V}(F) \backslash \mathcal{V}\left(F^{\prime}\right)\right| \leq 2$ for every facet $F^{\prime}$ of $F$. Otherwise, we could show with a similar calculation as before that there is a ridge $F^{\prime}$ of $P$ with a simplex face of dimension at least $d-\gamma+2$. This implies that $m=\gamma \geq 2$.

By Lemma 3.1, we have $k(F) \leq k(P)$. On the other hand,

$$
k(F) \geq\left\lfloor\frac{(d-1)-(\gamma-2)+1}{2}\right\rfloor=\left\lfloor\frac{d-\gamma+2}{2}\right\rfloor=k(P),
$$

by Lemma 3.3, since $d-\gamma+2$ is odd. Furthermore, $(d-1)-\gamma(F)=d-1-(\gamma-2)=$ $d-\gamma+1$ is even. Thus applying (i) yields that $F$ is of type $\mathcal{G}\left(n^{\prime}, m^{\prime}\right)$ with $n^{\prime}=d-3 \gamma+6$ and $m^{\prime}=\gamma-2$, as

$$
\operatorname{dim}\left(\mathcal{G}\left(n^{\prime}, m^{\prime}\right)\right)=n^{\prime}-1+3 m^{\prime}=d-3 \gamma+5+3 \gamma-6=d-1 .
$$

To get the $d-1$ different combinatorial types we modify $\mathcal{G}(n, m)$. Let $v_{0}, v_{1}$ be the vertices of one of the missing edges of one of the quadrilaterals. Let $Q$ be the convex hull of $\left(\mathcal{V}(\mathcal{G}(n, m)) \backslash\left\{v_{0}, v_{1}\right\}\right)$. Let $F$ be a $k$-face of $Q$ that has a quadrilateral 2-face, for $2 \leq k \leq d$. Such a face exists since $m=\gamma \geq 2$. Take the subdirect sum $R:=$ $(Q, F) \oplus\left(\Delta_{1}, \Delta_{1}\right)$. Then the $k$-face $F \oplus \Delta_{1}$ lies in a facet of $R$ whose complement has size 3 , as $F \oplus \Delta_{1}$ has a $(k-1)$-face that has a complement of size 3 in $F \oplus \Delta_{1}$.

For $p=f_{0}$ and $q=2 m$, the polytope $\mathcal{G}(n, m)$ has linkedness

$$
k(\mathcal{G}(n, m))=\left\lfloor\frac{p-q}{2}\right\rfloor=\left\lfloor\frac{d-\gamma+1}{2}\right\rfloor .
$$

This implies that $p \geq 3 q-2$ by Lemma 3.4.

Since $d-\gamma+1$ is even, also $p-q$ is even. The constructed polytope has $p$ vertices and exactly $q-1$ disjoint missing edges. Lemma 3.4 implies that the linkedness of $G(R)$ is

$$
\left\lfloor\frac{p-(q-1)}{2}\right\rfloor=\left\lfloor\frac{p-q}{2}\right\rfloor=k(\mathcal{G}(n, m)),
$$

since $p-q+1$ is odd and $p \geq 3(q-1)-1$.

See Figure 1 on the next page for minimal linkedness of polytopes in dimension 25 (this dimension was only chosen, because it is large enough to illustrate all interesting effects). 


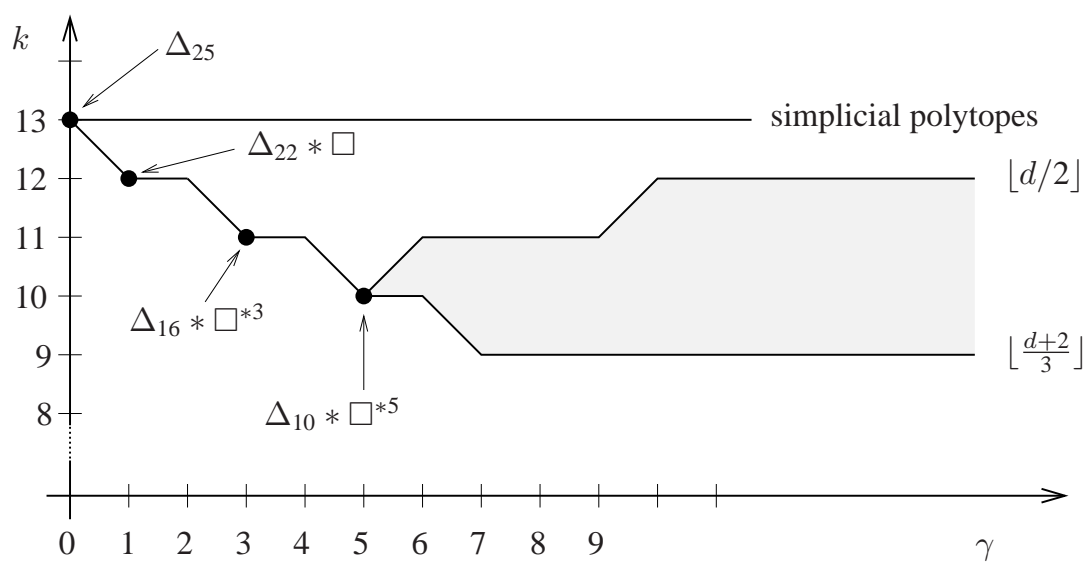

Figure 1. Linkedness of polytopes in dimension 25. Shown in this graph are (a) the minimal linkedness of simplicial polytopes, (b) the minimal linkedness of polytopes on few vertices (in dimension 25 known up to $\gamma=5$ ), (c) the unique extremal examples for $\gamma=0,1,3,5$, and (d) the best known upper and lower bounds for general polytopes.

\section{Open problems}

Theorem 2.2 and Corollary 3.7 imply the values for the minimal linkedness $k(d)$ as displayed in Table 1.

\begin{tabular}{r|ccccccccccccccc}
$d$ & 1 & 2 & 3 & 4 & 5 & 6 & 7 & 8 & 9 & 10 & 11 & 12 & 13 & 14 & 15 \\
\hline$k(d)$ & 1 & 1 & 1 & 2 & 2 & 2,3 & 3 & 3 & 3,4 & 4 & 4,5 & 4,5 & 5 & 5,6 & $5,6,7$
\end{tabular}

Table 1. Possible values of $k(d)$ in dimensions $1 \leq d \leq 15$.

In particular, we obtain exact values in dimensions 7, 10, and 13. The value $k(8)=3$ follows from Larman \& Mani's old lower bound [12] and Gallivan's upper bound [4].

The value $k(6)$ is the first open value of $k(d)$ and it seems to be a difficult problem to determine it. Our analysis of polytopes with few vertices in Theorem 3.11 shows that $k(6,0)=k(6,1)=k(6,2)=3$. We have also verified enumeratively that $k(6,3)=3$; beyond that we do not know much.

Problem 1. Determine $k(6)$ : Either show that all 6-polytopes are 3-linked, or give an example of a 6-polytope $P$ with $k(P)=2$.

Thomas \& Wollan [19] have shown that every 6-connected graph on $f_{0}$ vertices with at least $5 f_{0}-14$ edges is 3 -linked. This implies that all 6-polytopes that have only triangular faces are 3-linked, according to Kalai's Lower Bound Theorem [8].

Bereitgestellt von | Technische Universität Berlin 


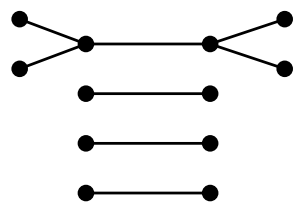

Figure 2. Is this a subgraph of the complement graph of some 8-polytope on 12 vertices?

One can construct polytopes with $f_{0}=3\lfloor d / 2\rfloor-1$ vertices that are not $\lfloor d / 2\rfloor$-linked. If $d$ is even let

$$
P:=\Delta_{2} * \square * \square * \underbrace{C_{3}^{\Delta} * \cdots * C_{3}^{\Delta}}_{m \text { factors }},
$$

where $C_{3}^{\Delta}$ denotes the 3-dimensional crosspolytope (polar to the cube $C_{3}$ ). Then $d=$ $4 m+8, f_{0}=6 m+11$ and $k(P)=2 m+3$. For $d$ odd let

$$
P:=\Delta_{4} * \square * \square * \square * \underbrace{C_{3}^{\Delta} * \cdots * C_{3}^{\Delta}}_{m \text { factors }} .
$$

Then $d=4 m+13, f_{0}=6 m+17$ and $k(P)=2 m+5$.

For large $f_{0}$, the known methods to prove upper bounds fail. This suggests the following question.

Problem 2. Are all $d$-polytopes on at least $3\lfloor d / 2\rfloor$ vertices $\lfloor d / 2\rfloor$-linked? Weaker: Is there some $N(d)$ such that every $d$-polytope on at least $N(d)$ vertices is $\lfloor d / 2\rfloor$-linked?

We only know of one obstruction for $d$-polytopes that prevents them from being $\lfloor d / 2\rfloor$-linked: The polytopes $\mathcal{G}(n, m)$ have many missing edges and not enough vertices to route all paths around the missing edges. If a polytope has $3\lfloor d / 2\rfloor$ or more vertices, there has to be a different obstruction if it is not $\lfloor d / 2\rfloor$-linked. Regarding Problem 2, it would be interesting to know if the graph in Figure 2 is a subgraph of the complement graph of an 8-polytope on 12 vertices. The complement of this graph is 8-connected and, for every vertex $v$, has a subdivision of $K_{9}$ rooted at $v$. However, it is not 4-linked.

\section{References}

[1] M. L. Balinski, On the graph structure of convex polyhedra in $n$-space. Pacific J. Math. 11 (1961), 431-434. MR0126765 (23 \#A4059) Zbl 0103.39602

[2] R. Diestel, Graph theory. Springer 2005. MR2159259 (2006e:05001) Zbl 1086.05001

[3] S. Gallivan, Properties of the one-skeleton of a convex body. PhD thesis, Univ. of London, 1974.

[4] S. Gallivan, Disjoint edge paths between given vertices of a convex polytope. J. Combin. Theory Ser. A 39 (1985), 112-115. MR787721 (86f:52013) Zbl 0572.05038

Bereitgestellt von | Technische Universität Berlin 
[5] B. Grünbaum, On the facial structure of convex polytopes. Bull. Amer. Math. Soc. 71 (1965), 559-560. MR0182966 (32 \#448) Zbl 0137.18002

[6] B. Grünbaum, Convex polytopes, second edition. Interscience Publ. 2003. MR1976856 (2004b:52001) Zbl 1024.52001

[7] H. A. Jung, Eine Verallgemeinerung des $n$-fachen Zusammenhangs für Graphen. Math. Ann. 187 (1970), 95-103. MR0268067 (42 \#2966) Zbl 0184.27601

[8] G. Kalai, Rigidity and the lower bound theorem. I. Invent. Math. 88 (1987), 125-151. MR877009 (88b:52014) Zbl 0624.52004

[9] G. Kalai, Some aspects of the combinatorial theory of convex polytopes. In: Polytopes: abstract, convex and computational (Scarborough, ON, 1993), vol. 440 of NATO Adv. Sci. Inst. Ser. C Math. Phys. Sci., 205-229, Kluwer 1994. MR1322063 (96b:52018) Zbl 0804.52006

[10] G. Kalai, Polytope skeletons and paths. In: Handbook of discrete and computational geometry, second edition, 455-476, CRC Press 2004. MR1730173 Zbl 0910.52005

[11] D. G. Larman, personal communication, 2007.

[12] D. G. Larman, P. Mani, On the existence of certain configurations within graphs and the 1skeletons of polytopes. Proc. London Math. Soc. (3) 20 (1970), 144-160.

MR0263687 (41 \#8288) Zbl 0201.56801

[13] D. A. Marcus, Simplicial faces and sections of a convex polytope. Mathematika 29 (1982), 119-127. MR673512 (84a:52011) Zbl 0502.52008

[14] P. McMullen, Constructions for projectively unique polytopes. Discrete Math. 14 (1976), 347358. MR0397552 (53 \#1411) Zbl 0319.52010

[15] P. McMullen, Transforms, diagrams and representations. In: Contributions to geometry (Proc. Geom. Sympos., Siegen, 1978), 92-130, Birkhäuser 1979. MR568496 (81i:52007) Zbl 0445.52006

[16] N. Robertson, P. D. Seymour, Graph minors. XIII. The disjoint paths problem. J. Combin. Theory Ser. B 63 (1995), 65-110. MR1309358 (97b:05088) Zbl 0823.05038

[17] P. D. Seymour, Disjoint paths in graphs. Discrete Math. 29 (1980), 293-309. MR560773 (82b:05091) Zbl 0457.05043

[18] Y. Shiloach, A polynomial solution to the undirected two paths problem. J. Assoc. Comput. Mach. 27 (1980), 445-456. MR581977 (82a:05061) Zbl 0475.68042

[19] R. Thomas, P. Wollan, An improved linear edge bound for graph linkages. European J. Combin. 26 (2005), 309-324. MR2116174 (2005h:05122) Zbl 1056.05091

[20] C. Thomassen, 2-linked graphs. European J. Combin. 1 (1980), 371-378. MR595938 (82c:05086) Zbl 0457.05044

[21] G. M. Ziegler, Lectures on polytopes. Springer 1995. MR1311028 (96a:52011) Zbl 0823.52002

Received 14 April, 2009; revised 28 July, 2009

A. Werner, Zuse-Institut Berlin, Takustr. 7, 14195 Berlin, Germany

Email: werner@zib.de

R. F. Wotzlaw, MA 6-2, Institute of Mathematics, Technische Universität Berlin,

Straße des 17. Juni 136, 10623 Berlin, Germany

Email: wotzlaw@math.tu-berlin.de 